\title{
Tunnel ionization of deep impurities in semiconductors induced by terahertz electric fields
}

\author{
S.D. Ganichev ${ }^{\mathrm{a}, \mathrm{b}, *}$ \\ anstitut für Exp. und Angew. Physik, Universität Regensburg, 93040 Regensburg, Germany \\ ${ }^{\mathrm{b}}$ A.F. Ioffe Physicotechnical Institute of the RAS, 194021 St. Petersburg, Russia
}

\begin{abstract}
An analysis is made of the ionization of deep impurity centers induced by high-intensity terahertz radiation whose photon energies are tens of times lower than the impurity ionization energy. Under these conditions, ionization can be described as phonon-assisted tunneling in which carrier emission is accompanied by defect tunneling in configuration space and electron tunneling in the electric field of the radiation. At high intensities the ionization is caused by direct tunneling. Within a broad range of intensity, frequency and temperature, the terahertz electric field of the radiation acts like a static field. For very high frequencies and low temperatures an enhancement in tunneling as compared to static fields takes place. (C) 1999 Elsevier Science B.V. All rights reserved.
\end{abstract}

Keywords: Tunnel ionization; Terahertz; Far-infrared; Deep centers

\section{Introduction}

The effect of high-frequency coherent radiation on tunneling in semiconductor superlattices and nanostructures has recently attracted considerable attention. The superposition of a static electric field and an alternating field causes a wealth of new phenomena as a result of photon assisted tunneling [1-3]. In all these cases tunneling is accomplished by a static electric field while the radiation influences the barrier penetration probability. An intense radiation field, however, can generate both the tunneling barrier and initiate tunneling. Such a tunneling process has been observed for deep impurities in semiconductors [4-6]. In contrast to tunneling ionization of atoms, where only electron tunneling takes place [7], phonon-assisted tunneling ionization of impurities in solids is accomplished by two simultaneous tunneling

\footnotetext{
* Correspondence address: Institut für Exp. und Angew. Physik, Universität Regensburg, D-93040 Regensburg, Germany. Tel.: + 49-941-943-2050; fax: + 49-941-943-4223.

E-mail address: sergey.ganichev@physik.uni-regensburg.de (S.D. Ganichev)
}

processes, electron tunneling and the redistribution of the vibrational system by defect tunneling [8,9]. With increasing electric field strength direct carrier tunneling from the ground state into continuum, without participation of phonons, becomes dominant.

\section{Theoretical consideration}

In most cases deep impurities have one bound state which phenomenologically can be approximated by a potential well. The emission and capture of electrons or holes by deep impurities in semiconductors can be considered in the adiabatic approximation. Due to strong electron-phonon interaction a system consisting of local impurity vibrations and an electron is characterized by two adiabatic potentials $U_{1}(x)$ and $U_{2}(x)$ as a function of a configuration coordinate $x$ (see insets in Fig. 3) [8,10-13]. Following the Huang-Rhys model [10] these two adiabatic potentials correspond to the free electron bound to the impurity and to a free electron with zero kinetic energy, respectively. The energy separation between the minima of $U_{1}$ and $U_{2}$ is the thermal ionization energy $\varepsilon_{\mathrm{T}}$ of the electron. Thermal emission of carriers from the bound state into the continuum is accomplished 
a)

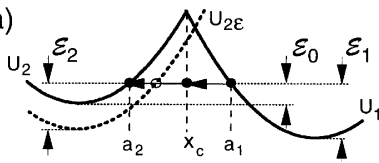

b)

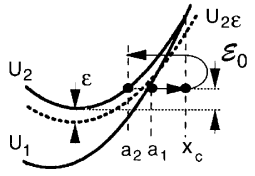

Fig. 1. Tunneling trajectories for the ionization of autolocalized (a) and substitutional (b) deep impurities.

by (i) thermal excitation of the system in the adiabatic bound state potential $U_{1}$ by a vibrational energy $\mathscr{E}_{1}$ and (ii) tunneling of the impurity configuration from this state into an excited state of the ionized configuration $U_{2}$ with energy $\mathscr{E}_{2}$ (Fig. 1) [8,9,14-16].

In the presence of an electric field the electron can be emitted at a negative kinetic energy $-\varepsilon$ due to tunneling through the triangular potential barrier formed by the potential well and the electric field (see inset in Fig. 4). Hence, the adiabatic potential of the ionized configuration $U_{2}$ is shifted to a lower energy $U_{2 \varepsilon} \equiv U_{2}-\varepsilon$ (Figs. 1 and 3). Thus electron emission in an electric field is achieved by two tunneling processes, electron tunneling with probability $P_{\mathrm{e}}$ and tunneling of the defect from the adiabatic potential $U_{1}(x)$ to potential $U_{2 \varepsilon}$ with the probability $P_{\mathrm{d}}[8,9,14-16]$.

The ionization probability may be written as

$e(E)=\iint P_{\mathrm{e}} P_{\mathrm{d}} \exp \left(-\mathscr{E}_{1} / k_{\mathrm{B}} T\right) \mathrm{d} \varepsilon \mathrm{d} \mathscr{E}_{1}$.

The Boltzmann factor takes into account the thermal excitation of the system in the adiabatic potential $U_{1}$.

In the semi-classical approximation, neglecting preexponential factors, the defect tunneling probability is given by $[8,15,16]$

$P_{\mathrm{d}} \propto \exp \left(-2\left(S_{2} \mp S_{1}\right)\right)$,

$$
S_{i}\left(\mathscr{E}_{i}\right)=\frac{\sqrt{2 M}}{\hbar} \int_{a_{i}}^{x_{\mathrm{c}}} \mathrm{d} x \sqrt{U_{i}(x)-\mathscr{E}_{i}}, \quad i=1,2,
$$

where $M$ is a mass corresponding to the mode of impurity vibration, $a_{i}$ and $x_{\mathrm{c}}$ are shown in Fig. 1, and the minus and plus sign are related to the particular impurity configuration shown in Fig. 1a and b, respectively.

Electron tunneling in an alternating electric field $E=E_{0} \cos (\omega t)$ has been treated theoretically by Keldysh [7] (see also [17]). The tunneling probability is given by

$P_{\mathrm{e}} \propto \exp \left(-2 S_{\mathrm{e}}(\varepsilon)\right)$,

$$
S_{\mathrm{e}}(\varepsilon)=-\frac{\varepsilon}{\hbar} \int_{0}^{\tau_{\mathrm{e}}} \frac{1}{\gamma^{2}} \sinh ^{2}(\omega \tau) \mathrm{d} \tau+\frac{\varepsilon \tau_{\mathrm{e}}}{\hbar} .
$$

Here $\gamma \equiv \sqrt{2 m^{*} \varepsilon} \omega / e E$ and $\sinh \left(\omega \tau_{\mathrm{e}}\right)=\gamma ; m^{*}$ and $e$ are the electron effective mass and charge, respectively, and

$\tau_{\mathrm{e}}=\hbar \partial S_{\mathrm{e}} / \partial \varepsilon$ has the meaning of an electron tunneling time $[18,19]$.

The integral in Eq. (1) has been calculated using the saddle point method [6]. In the case of weak electric fields, i.e., as long as the saddle point energy $\varepsilon \ll \varepsilon_{\mathrm{T}}$, the exponent can be expanded into a power series of $\varepsilon$. Taken into account that $\mathscr{E}_{2}=\mathscr{E}_{1}-\left(\varepsilon_{\mathrm{T}}-\varepsilon\right)$ obtains

$$
\begin{gathered}
\left.S_{2} \mp S_{1} \simeq\left(S_{2} \mp S_{1}\right)\right|_{\varepsilon=0}-\frac{\varepsilon \tau_{2}}{\hbar} \\
\text { with } \tau_{2}=\left.\frac{\hbar \partial\left|S_{2}\right|}{\partial \mathscr{E}_{2}}\right|_{\varepsilon=0} .
\end{gathered}
$$

Then the saddle point condition gives

$\tau_{2}=\tau_{\mathrm{e}}, \quad \tau_{2}=\frac{\hbar}{2 k_{\mathrm{B}} T} \pm \tau_{1}$,

where $\tau_{1}=\hbar \partial\left|S_{1}\right| /\left.\partial \mathscr{E}_{1}\right|_{\varepsilon=0}$. The first condition in Eq. (5) states that the electron tunneling time $\tau_{\mathrm{e}}$ is equal to the defect tunneling time $\tau_{2}$ for tunneling under the potential $U_{2}$.

Finally, the ionization probability of phonon-assisted tunneling is given by [6]

$e(E) \propto \exp \left[\frac{E^{2}}{E_{\mathrm{c}}^{* 2}}\right]=\exp \left[\frac{E^{2} e^{2}\left(\tau_{2}^{*}\right)^{3}}{3 m^{*} \hbar}\right]$,

$\left(\tau_{2}^{*}\right)^{3}=\frac{3}{4 \omega^{3}}\left(\sinh \left(2 \omega \tau_{2}\right)-2 \omega \tau_{2}\right)$.

This result shows that $\left(\tau_{2}^{*}\right)^{3}$ increases exponentially as a function of $\omega \tau_{2}$. As the ionization probability itself depends exponentially on the third power of $\tau_{2}^{*}$ the tunneling ionization is drastically enhanced with rising $\omega \tau_{2}$. This can be achieved not only by increasing the radiation frequency $\omega$ but also by lowering the temperature (Eq. (5)). In the limit $\omega \tau_{2} \ll 1$ the time constant $\tau$ 㐘 approaches the tunneling time $\tau_{2}$ giving the same result as the static field regime obtained in Ref. [16].

\section{Samples and experimental technique}

The tunneling ionization of deep impurities by highintensity FIR radiation with photon energies much smaller than the thermal impurity ionization energy $\varepsilon_{\mathrm{T}}$ has been studied for different types of deep impurities: substitutional impurities with weak electron-phonon coupling (Ge:Au, Ge:Hg, Ge:Cu, Ge:Zn, Si:Au, $\mathrm{GaP}: \mathrm{Te}$ with acceptor concentrations in the range $10^{14}-10^{15} \mathrm{~cm}^{-3}$ ) and autolocalized $\mathrm{DX}^{-}$centers with strong electron-phonon coupling $\left(\mathrm{Al}_{x} \mathrm{Ga}_{1-x} \mathrm{As}: \mathrm{Te}\right.$, $\mathrm{Al}_{x} \mathrm{Ga}_{1-x} \mathrm{Sb}$ :Te with $x=0.35$ and 0.5 ). In the investigated temperature range from 4.2 to $80 \mathrm{~K}$ or for autolocalized impurities up to $150 \mathrm{~K}$, practically all impurities are occupied in thermal equilibrium. 
The change of the ionization probability in the electric field which is proportional to the change in the free carrier concentration can be detected as photoconductive signal under FIR-laser illumination. The samples were placed in a temperature-controlled optical cryostat. Penetration of light in the medium IR range into the cryostat was prevented by the use of crystalline quartz filters, and in the visible, with a $1 \mathrm{~mm}$-thick black-polyethylene filter. Measurements have been carried out using a standard $50 \Omega$ load resistor circuit, taking care that the bias voltage across the sample was substantially below the threshold of electric breakdown, which for bulk samples is at about $5 \mathrm{~V} / \mathrm{cm}$.

The radiation sources used was a pulsed far-infrared molecular laser optically pumped by a TEA- $\mathrm{CO}_{2}$ laser $[20,21]$. The $\mathrm{CO}_{2}$ laser has been used for optical pumping because of its wavelength range from 9.2-10.6 $\mu \mathrm{m}$, which includes strong vibrational-rotational absorption lines of many molecules. Commercial TEA- $\mathrm{CO}_{2}$ lasers (URENKO-204) offer high stability and high power for this purpose. More than 10 strong single lines in the terahertz range from 3.78 to $68 \mathrm{THz}$ (corresponding wavelengths are from 496 to $35 \mu \mathrm{m}$ ) have been obtained using $\mathrm{NH}_{3}, \mathrm{D}_{2} \mathrm{O}$ and $\mathrm{CH}_{3} \mathrm{~F}$ as the active gases for optically pumped laser [5]. The radiation pulse length varies for different lines from 10 to $100 \mathrm{~ns}$. The radiation is focused to a spot of about $1 \mathrm{~mm}^{2}$, with the maximum intensity reaching as high as $5 \mathrm{MW} / \mathrm{cm}^{2}$ corresponding to electric fields up to about $50 \mathrm{kV} / \mathrm{cm}$. The intensity, pulse shape and the spatial distribution of the laser radiation were measured with fast room temperature photodetectors based on the photon drag effect [22], intraband $\mu$-photoconductivity [23] and the Spirikon pyroelectric camera, respectively.

Ionization of deep impurities by far infrared radiation has been observed for all samples in the whole frequency range investigated. A photoconductive signal increasing nonlinearly with incident power $\left(I \leqslant 5 \mathrm{MW} / \mathrm{cm}^{2}\right.$, $E \leqslant 5 \times 10^{4} \mathrm{~V} / \mathrm{cm}$ ) has been observed in spite of the fact that the photon energies were much smaller than the binding energy of the impurities. Ionization can be attributed to phonon-assisted and direct tunneling over a wide range of temperature, frequency and electric field strength [5].

At not too low temperatures and not too high frequencies the tunneling probability is independent of frequency and exponentially increases with the square of the electric field strength ${ }^{1}$ (see Eq. (6)). This is illustrated in Fig. 2a

\footnotetext{
${ }^{1}$ In the case of charged impurities (all substitutional impurities investigated) a deviation from the field dependence $e(E) \propto \exp \left(E^{2} / E_{\mathrm{c}}^{* 2}\right)$ can be seen in relatively low-field strength (up to $1 \mathrm{kV} / \mathrm{cm}$ ) where the defects are thermally ionized through the Poole-Frenkel effect and the ionization probability is proportional to $e(E) \propto \exp \left(\sqrt{\left(Z e^{3} E / \kappa\right)} / k_{\mathrm{B}} T\right)[5,24,25]$.
}

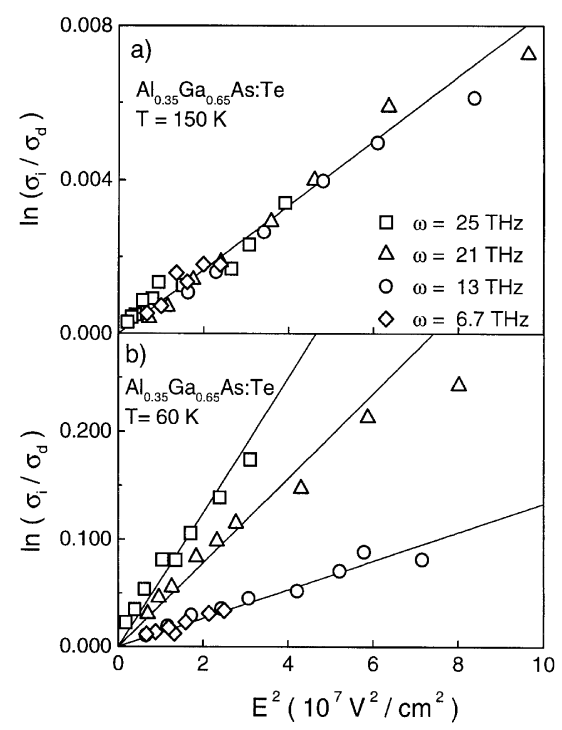

Fig. 2. The ionization probability (given by the ratio of the conductivity under illumination and in the dark $\sigma_{\mathrm{i}} / \sigma_{\mathrm{d}}$ ) of $\mathrm{Al}_{0.35} \mathrm{Ga}_{0.65} \mathrm{As}: \mathrm{Te}$ as a function of the square of the electric field of the radiation. Lines shows $\propto \exp \left(E^{2} / E_{\mathrm{c}}^{* 2}\right)$.

where experimental results obtained with $\mathrm{AlGaAs}$ : Te at $T=150 \mathrm{~K}$ are shown. In this quasi-static regime the electron tunnels at the instantaneous magnitude of the electric field in a time shorter than the period of oscillation and thus the electric field acts like a static field. Such a behaviour has been observed for all materials at sufficiently high temperatures. In this quasi-static regime [5] the characteristic field, which can be determined experimentally, is given by $E_{\mathrm{c}}^{* 2}=\left(3 m^{*} \hbar\right) /\left(\tau_{2}^{* 3} e^{2}\right)$ with $\tau_{2}^{*}=\tau_{2}$. Thus, the investigation of field dependence of ionization probability allows one to determine the defect tunneling time $\tau_{2}$ [26]. Fig. 3 presents the temperature dependence of the tunneling time $\tau_{2}$ obtained for various samples. For the purpose of comparison, $\hbar / 2 k_{\mathrm{B}} T$ is also plotted in Fig. 3. Clearly, $\tau_{2}$ is larger than $\hbar / 2 k_{\mathrm{B}} T$ for substitutional impurities and smaller than $\hbar / 2 k_{\mathrm{B}} T$ for the DX-centers. Thus, the tunneling time reflects the structure of the potential barriers which is distinct for the two potential configurations discussed here. The phonon-assisted ionization of deep impurities in semiconductors by contactless application of short pulses of terahertz radiation has been proposed as a method for the characterization of defects $[5,26,30]$. The field dependence of the signal allows one to determine the defect tunneling times, the Huang-Rhys parameter and the basic structure of the adiabatic defect potentials. The Poole-Frenkel effect, which can be observed for charged impurities only (see footnote 1), can be used to determine the defect charge state.

This frequency independent tunneling is limited to frequencies $\omega$ with $\omega \tau_{2}<1$ (Eq. (7)). The enhancement of 


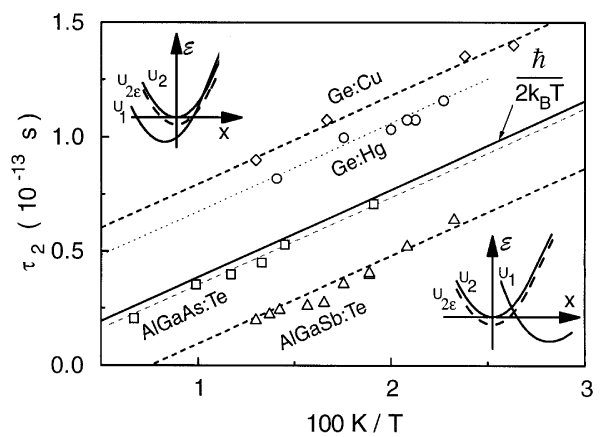

Fig. 3. Tunneling times $\tau_{2}$ as a function of $1 / T$ for different samples. The full line shows $\hbar / 2 k_{\mathrm{B}} T$, the broken lines are plotted according to Eq. (5). Inset: Adiabatic potentials for substitutional impurities (top left) and autolocalized impurities (bottom right).

tunneling at frequencies higher than the reciprocal tunneling time has been anticipated in a number of theoretical papers [7,17,27-29], but has been demonstrated experimentally only recently [6]. In contrast to static electric fields where the electron tunnels at a fixed energy, the energy of the electron is not conserved during tunneling in alternating fields (see inset in Fig. 4). In this case the electron can absorb energy from the field, which leads to a sharp increase of the tunneling probability with increasing frequency. This effect has been observed for various impurities in different semiconductors and is demonstrated for AlGaAs: Te in Fig. 2b. For the case of $\omega \tau_{2} \geqslant 1$ the ionization probability still depends exponentially on the square of the electric field strength but the characteristic field becomes frequency dependent and ionization is drastically enhanced with rising frequency.

In Fig. 4 the ratio $\tau_{2} / \tau_{2}$ calculated from Eq. (7) is plotted as a function of $\omega \tau_{2}$ and compared to experimental results obtained from the measured values of $E_{\mathrm{c}}^{*}$ for various the frequencies, temperatures and materials. The tunneling times $\tau_{2}$ were determined from frequency independent values of $E_{\mathrm{c}}^{*}$. The experimental results shown in Fig. 4 are grouped according to the materials. For each material the variation of the value of $\omega \tau_{2}$ has been obtained by applying different radiation frequencies in the range from 6.7 to $25 \mathrm{THz}$ and different temperatures between 20 and $150 \mathrm{~K}$. It should be pointed out that the theory leading to good agreement with experiment does not contain any fitting parameters.

Further decrease of the temperature leads to a much stronger frequency dependence of the ionization probability. Fig. 5 shows experimental results for $\mathrm{Ge}: \mathrm{Cu}$ at $T=4.2 \mathrm{~K}$ in the frequency range between 3.4 and 25 Thz. In order to display in one figure the total set of data covering eight order of magnitude in the square of the

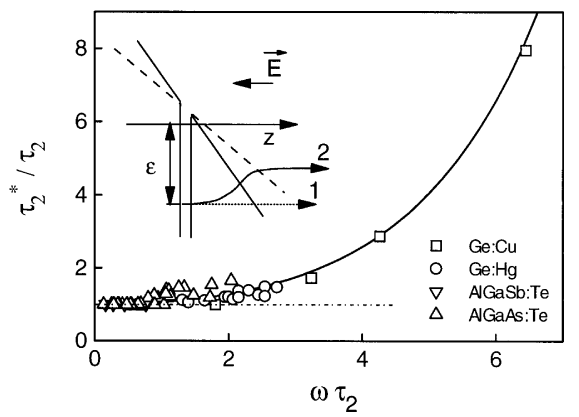

Fig. 4. Ratio $\tau_{2}^{*} / \tau_{2}$ as a function of $\omega \tau_{2}$. The line shows the dependence according to Eq. (7) Inset: Electron tunneling trajectory: (1) in a static electric field, and (2) in an alternating field.

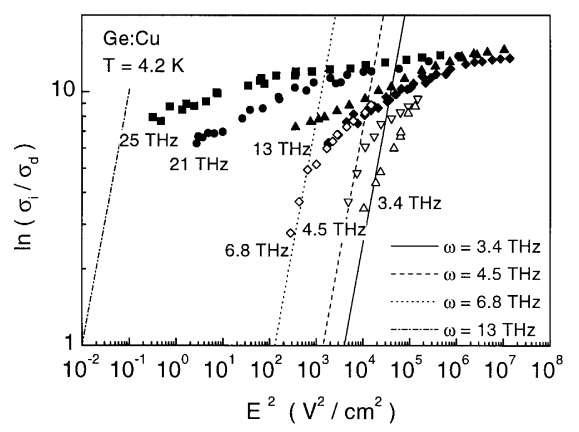

Fig. 5. The ionization probability (given by the ratio of the conductivity under illumination and in the dark $\sigma_{\mathrm{i}} / \sigma_{\mathrm{d}}$ ) of $\mathrm{Ge}: \mathrm{Cu}$ at $4.2 \mathrm{~K}$ as a function of the square of the electric field of the radiation. Solid symbols are obtained with molecular optically pumped FIR laser and open symbols with Santa Barbara FreeElectron-Laser [6]. Lines show $\propto \exp \left(E^{2} / E_{\mathrm{c}}^{* 2}\right)$ for four lowest frequencies used in experiment.

electric field, $\log \left(E^{2}\right)$ has been plotted on the abscissa. To make a easy comparison to the $\exp \left(E^{2} / E_{\mathrm{c}}^{2}\right)$ dependence of $\sigma_{\mathrm{i}} / \sigma_{\mathrm{d}}$ possible, a $\log \log$ presentation has been used for the ordinate.

At $4.2 \mathrm{~K}$ the condition $\omega \tau_{2} \gg 1$ is valid for whole frequency range investigated here. The results in Fig. 5 show that at liquid helium temperature in the limit of $\omega \tau_{2} \gg 1$ a drastic frequency dependence is observed. For a given constant signal a change of six orders of magnitude in electric fields squared needs only a factor of seven change in frequency. Note, that this effect is observed only for relatively low fields. In Fig. 5 the results of calculations for phonon-assisted tunneling with $\tau_{2}$ determined at higher temperatures is plotted. It is seen that for the three lowest frequencies the experimental points at low field strength are reasonably well described by the emission probabilities of phonon-assisted tunneling calculated from Eqs. (6) and (7). However, for higher frequencies the calculated field dependence of 


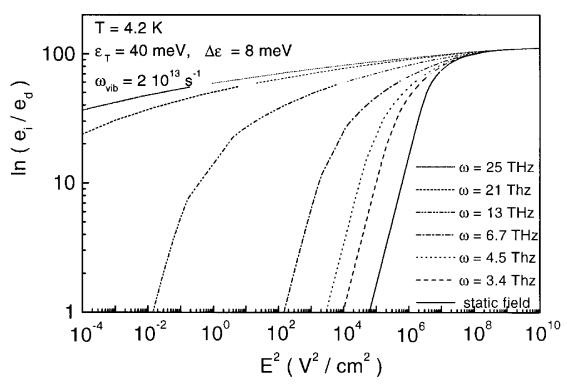

Fig. 6. Field dependence of ionization probability calculated after Eqs. (1)-(3) for all values of the energy of electron tunneling $\varepsilon$ for $\mathrm{Ge}: \mathrm{Cu}$ at $4.2 \mathrm{~K}$ and frequencies used in the experiments taking into account phonon-assisted and direct tunneling but ignoring Coulomb interaction.

phonon-assisted tunneling does not fit the experimental results in the whole range of electric fields where a signal could be detected. Furthemore, at higher field strengths the measured field dependence of the emission probability is much weaker than the theoretical result and the frequency dependence practically disappears. Similar results have been obtained for autolocalized DX-centers in AlGaAs:Te [31].

This complex dependence of ionization probability on field strength and radiation frequency is a result of the transition from phonon-assisted tunneling at low-field strength to direct tunneling without phonons at high fields. The emission probability for phonon-assisted tunneling as a function of the electric field strength given by Eq. (6) was obtained in the limit that corrections to thermal emission resulting from electron tunneling are small, i.e., the energy of electron tunneling $\varepsilon$ is much smaller than the defect tunneling energy $\mathscr{E}_{0}$ and the energy of thermal ionization $\varepsilon_{\mathrm{T}}$. In the opposite limit, $\varepsilon>\varepsilon_{\mathrm{T}}, \mathscr{E}_{0}$, direct carrier tunneling from the ground state into the continuum, without participation of phonons, becomes dominant [5]. Direct electron tunneling occurs at the crossing of the $U_{2 \varepsilon}$ and $U_{1}$ potential curves, where an electronic transition is possible without any change in the configuration coordinate. This effect, leading to weaker field dependence of the ionization probability in comparison to that of phonon-assisted tunneling dominates the ionization process at very high fields.

Fig. 6 shows the result of calculations based on Eqs. (1)-(3) for all values of the electron tunneling energy $\varepsilon$ for $\mathrm{Ge}: \mathrm{Cu}$ at $4.2 \mathrm{~K}$ and frequencies used in the experiments taking into account both processes but ignoring Coulomb interaction. It is seen that the theory describes well the experimentally observed features of the field and frequency dependence of tunneling ionization. The disappearence of frequency dependence of the ionization probability at high-field strengths is caused by a decrease in the tunneling time $\tau_{2}$ with rising electric field strength resulting finally in $\omega \tau_{2}$ less than unity. This is due to the fact that at high-field strength defect tunneling occurs at energies much smaller than the energy of phonon-assisted tunneling which changes the tunneling trajectory. To achieve the quantitative agreement one needs to improve the theory by taking into account the charge of the impurities.

Finally, all our measurements have been carried out with deep impurities in semiconductors, however, because tunneling is crucial in numerous processes in physics, chemistry, and biology we expect that an enhancement of tunneling induced by application of high-intensity coherent radiation will have significant consequences.

\section{Acknowledgements}

The author would like to thank W. Prettl, I.N. Yassievich, V.I. Perel and E. Ziemann for active help and discussion. Financial support by the DFG and the RFFI is gratefully acknowledged.

\section{References}

[1] P.S.S. Guimaraes, B.J. Keay, J.P. Kaminski, S.J. Allen Jr., P.F. Hopkins, A.C. Gossard, L.T. Florez, J.P. Harbinson, Phys. Rev. Lett. 70 (1993) 3792.

[2] B.J. Keay, S.J. Allen Jr., J. Gallan, J.P. Kaminski, K.L. Campman, A.C. Gossard, U. Bhattacharya, J.W. Rodwell, Phys. Rev. Lett. 75 (1995) 4098.

[3] C.J.G.M. Langerak, B.N. Murdin, B.E. Cole, J.M. Chamberlain, M. Henini, M. Pate, G. Hill, Appl. Phys. Lett. 67 (1995) 3453.

[4] S.D. Ganichev, W. Prettl, P.G. Huggard, Phys. Rev. Lett. 71 (1993) 3882.

[5] S.D. Ganichev, W. Prettl, I.N. Yassievich, Rev. Phys. Solid State 39 (1997) 1703.

[6] S.D. Ganichev, E. Ziemann, Th. Gleim, W. Prettl, I.N. Yassievich, V.I. Perel, I. Wilke, E.E. Haller, Phys. Rev. Lett. 80 (1998) 2409.

[7] L.V. Keldysh, Sov. Phys. JETP 20 (1965) 1307.

[8] V.N. Abakumov, V.I. Perel, I.N. Yassievich, Nonradiative Recombination in Semiconductors, in: V.M. Agranovich, A.A. Maradudin (Eds.), Modern Problems in Condensed Matter Sciences, Vol. 33, North-Holland, Amsterdam, 1991.

[9] P.T. Landsberg, Recombination in Semiconductors, Cambridge University Press, New York, 1991.

[10] K. Huang, A. Rhys, Proc. Roy. Soc. London, Ser. A 204 (1950) 406.

[11] C.H. Henry, D.V. Lang, Phys. Rev. B 15 (1977) 989.

[12] P.M. Mooney, T.N. Theis, Comments Condens. Matter Phys. 16 (1992) 167.

[13] R.C. Newman, Semicond. Sci. Technol. 9 (1994) 1749.

[14] S. Makram-Ebeid, M. Lannoo, Phys. Rev. B 25 (1982) 6406.

[15] T. Markvart, J. Phys. C 17 (1984) 6303.

[16] V. Karpus, V.I. Perel, Sov. Phys. JETP 64 (1986) 1376. 
[17] L.D. Landau, E.M. Livshitz, Quantum Mechanics, Pergamon, Oxford, 1977, p. 287.

[18] M. Büttiker, R. Landauer, Phys. Rev. Lett. 49 (1982) 1739.

[19] R. Landauer, Th. Martin, Rev. Mod. Phys. 66 (1994) 217.

[20] T.Y. Chang, T.J. Bridges, Opt. Commun. 1 (1970) 423.

[21] Th. de Temple, in: K.J. Button (Ed.), Infrared and Millimeter Waves, Vol. 1, 1979, p. 129.

[22] S.D. Ganichev, Ya.V. Terent'ev, I.D. Yaroshetskiı̌, Sov. Tech. Phys. Lett. 11 (1985) 20.

[23] S.D. Ganichev, S.A. Emel'yanov, A.G. Pakhomov, Ya.V. Terent'ev, I.D. Yaroshetskiŭ, Sov. Tech. Phys. Lett. 11 (1985) 377.

[24] S.D. Ganichev, J. Diener, I.N. Yassievich, W. Prettl, Europhys. Lett. 29 (1995) 315.

[25] S.D. Ganichev, E. Ziemann, W. Prettl, A. Istratov, E.R. Weber, MRS Symposium. Proceedings, San Francisco, 1999, to be published.
[26] S.D. Ganichev, J. Diener, I.N. Yassievich, W. Prettl, B.K. Meyer, K.W. Benz, Phys. Rev. Lett. 75 (1995) 1590.

[27] Yu.A. Bychkov, A.M. Dykhne, Sov. Phys. JETP 31 (1970) 928.

[28] B.I. Ivlev, V.I. Mel'nikov, Phys. Rev. Lett. 55 (1985) 1614.

[29] M.V. Ammosov, N.B. Delone, V.P. Krainov, Sov. Phys. JETP 64 (1986) 1191.

[30] E. Ziemann, S.D. Ganichev, I.N. Yassievich, K. Schmalz, W. Prettl, in: S. Ashok, J. Chevallier, K. Sumino, B.L. Sopori, W. Goetz (Eds.), Defect and Impurity Engineered Semiconductors and Devices II, Mater. Res. Soc. Symp. Proc. 510 (1998) 595.

[31] H. Ketterl, E. Ziemann, S.D. Ganichev, I.N. Yassievich, A. Belyaev, S. Schmult, W. Prettl, 1999, to be published. 